\title{
Systematic review for cryoablation of Barrett's esophagus: Can we draw conclusions by combining apples, oranges and a banana?
}

\section{(๑) $\odot \ominus$}

\author{
Authors \\ S. N. van Munster, Jacques Bergman, Roos E. Pouw
}

Institution

Amsterdam University Medical Centres, Gastroenterology \&

Hepatology, Amsterdam, Noord-Holland, Netherlands

Bibliography

DOI https://doi.org/10.1055/a-1096-0756 |

Endoscopy International Open 2020; 08: E465-E466

(c) Georg Thieme Verlag KG Stuttgart · New York

eISSN 2196-9736

\author{
Corresponding author \\ Jacques Bergman, Amsterdam University Medical Centres - \\ Gastroenterology \& Hepatology, Meibergdreef 9, 1105 AZ \\ Amsterdam, Netherlands \\ Fax: +0031205669000 \\ j.j.bergman@amsterdamumc.nl
}

Most guidelines for Barrett's esophagus (BE) recommend radiofrequency ablation (RFA) as first-choice option for dysplastic BE, or after endoscopic resection of early cancer. Numerous highquality (randomized) studies have demonstrated that RFA for these indications is efficient and safe $[1,2]$. In recent years, an increasing number of studies appeared, evaluating use of new ablation techniques such as cryospray ablation, cryoballoon ablation (CBA) and (hybrid) argon plasma coagulation (APC).

In the current issue of this journal, Westerveld et al report the results of a systematic review, aiming to report rates of complete eradication of intestinal metaplasia (CE-IM) and dysplasia (CE-D) after CBA. The meta-analysis included seven clinical studies with a total of 272 patients. The pooled rates for all outcomes were: feasibility (95.8\%), CE-IM (85.8\%) and CE-D $(93.8 \%)$, defined as the proportion of patients that achieved clearance of IM/dysplasia. The overall adverse event rate was $12.5 \%$. Based on these results, the authors conclude that CBA is a safe and effective ablation technique for treatment of $B E$ neoplasia.

A systematic review with meta-analysis can give a balanced point estimate of all available evidence, and is therefore considered the highest quality of evidence for a therapeutic study [3]. However, a good systematic review should include studies that address the same outcomes in comparable populations. For CBA, currently only a limited number of publications are available, mainly feasibility studies. In our opinion, a systematic review and meta-analysis at this time is therefore premature and inappropriate, as are the conclusions drawn by Westerveld et al.

This meta-analysis is mostly a case of comparing apples with oranges, and a banana. The banana in this case being the study by Canto et al, assessing outcomes of CBA for squamous dys- plasia. This study accounts for 120 of 272 cases in this meta-analysis which aims to assess outcomes of CBA for eradication of IM and dysplasia [4]. Squamous dysplasia in the esophagus is a totally different disease entity than dysplasia arising in BE. Inclusion of this study in this systematic review therefore makes no sense at all.

When looking at the apples and oranges, the remaining six studies (152 patients) were performed for eradication of $\mathrm{BE}$ with dysplasia. However, these studies answered different questions and reported different outcome measures. Five studies report outcomes after a single CBA treatment. An accurate outcome measure after a single treatment could be the BE surface regression-an endoscopic assessment of the percentage of the treatment area that converted to squamous epithelium at first follow-up. Three of these five studies were reported by our group, and we indeed reported the BE surface regression as primary outcome [5-7]. One should, however, not confuse this outcome of percentage of the BE segment eradicated per patient after one CBA session with rates for CE-IM and/or CE-D (e.g. the proportion of all patients that achieve CE-IM/D) after consecutive treatment sessions. Assessment of CE-IM and CE-D only makes sense after consecutive treatment sessions with the aim to eradicate the entire BE segment, yet these outcomes cannot be extracted from studies in which only a single CBA treatment was performed as was the case in five of the included studies.

Furthermore, it must be mentioned that most of the included studies were feasibility studies. The results of these studies cannot be extrapolated to the general BE population treated with ablative therapy. First, in most of the feasibility studies, only a relatively small surface area was treated, with CBA ap- 
plied either for a small BE island or short segment BE (median C0-2 M0-5). Furthermore, in some studies, patients were included after prior RFA treatment, not as refractory cases, but on the contrary as patients with good regression and without complications after RFA. Logically, these patients were likely to respond well to CBA as well. The results of these feasibility studies therefore give an unrealistically positive outcome for efficacy and underestimation of the complication risk. Whereas these feasibility studies are pivotal in the initial phase of a new ablation tool, the results should not be extrapolated as benchmarks for the technique in the general population.

If a systematic review and/or meta-analysis does not help us to answer what ablation technique we should use for BE ablation, then what do we need? When a technique has been shown to be feasible for conversion of BE to squamous mucosa in small series, without technical issues or major complications, the next step is to assess sufficient maturation of the technique. In our opinion this requires completion of at least one large (i.e. $>100$ patients) single-arm, prospective, multicenter study that shows that the technique is feasible and safe (i. e. serious complications in $<5 \%$ ). Such a study should be accompanied with "endoscopic tips and tricks" learned during this maturation phase. Once a new technique is evaluated in such a study, a logical next step might be to compare the new ablation tool to the current gold standard of RFA. Ideally, such a randomized study should be performed in centers with extensive experience in treatment of BE neoplasia, among a homogeneous study population with dysplastic BE, and after endoscopic resection of visible lesions, if present. After consecutive treatment sessions, the outcome of interest should be the proportion of patients with complete endoscopic eradication of $\mathrm{BE}$ and histologic confirmation by absence of IM. Given the high point-estimate for RFA in expert centers for this outcome of $>90 \%$, a non-inferiority study would probably be the best fitting design. We recently performed a power calculation for this imaginary trial and we showed that a total of 1,590 patients should be included [8].

Should we undertake such an enormous investment in terms of time, money and patients, which would give us, at best, an equally effective tool? Or would the outcomes of such a study, which would run for many years, be already outdated by the time the study has completed its follow-up? This study only appears useful if the new ablation technique really holds promise for significant ancillary benefits over RFA. In our opinion, it is questionable whether CBA or APC will provide such significant ancillary benefits. One of the suggested benefits of CBA is improved tolerability, but one may question whether this alone is sufficient. In none of the prospective RFA studies post-procedural pain has been reported as a serious issue. Lower costs have been suggested as a benefit for APC. However, this is purely based on the price of materials and costs-efficacy analysis has never been systematically studied. Given the additional time and treatment sessions required for eradication of a significant Barrett's segment with APC compared to RFA, the difference in costs may be less then generally assumed.

The authors mention that CBA might also be useful in patients that do not respond to RFA, i. e. RFA-refractory cases. In- deed, instead of focusing on a new tool to replace RFA, we might better explore whether these new techniques are complementary to our current multimodality treatment approach. Although our current approach of ER and RFA is very safe and very effective, we still have to deal with several challenges. Improvements in the current treatment algorithm for BE with dysplasia and early cancer could be found in new ablation tools that do work in RFA-refractory patients, that can be used as rescue treatment in patients with persisting lesions that cannot be removed with ER, or in treatment of patients with a stenosis that hampers passage of the RFA catheter.

\section{Conclusion}

In conclusion, a new ablation tool first needs several small studies to prove that it is feasible and these should be followed by at least one large, single-arm prospective study to prove that the technique is mature. Before that, systematic reviews do not provide reliable evidence for efficacy and safety of the technology.

\section{Competing interests}

Prof. Bergman has received financial support for clinical trials from Medtronic, Pentax Medical, C2 Therapeutics, Aqua Medical, Boston Scientific, Erbe Medical, Cernostics, Ninepoint Medical, Fujifilm, and Olympus. He is a recipient of speaker's fees from Fujifilm and is a consultant for Olympus and Fractyl.

References

[1] Qumseya B, Sultan S. ASGE Standards Of Practice Committee. et al. ASGE guideline on screening and surveillance of Barrett's esophagus. Gastrointest Endosc 2019; 90: 335-359 e2

[2] Weusten B, Bisschops R, Coron E et al. Endoscopic management of Barrett's esophagus: European Society of Gastrointestinal Endoscopy (ESGE) Position Statement. Endoscopy 2017; 49: 191-198

[3] Oxford Levels of Evidence Group. The Oxford 2011 Levels of Evidence. Oxford Centre for Evidence-Based Medicine; 2011

[4] Canto MI, Abrams JA, Kunzli HT et al. Nitrous oxide cryotherapy for treatment of esophageal squamous cell neoplasia: initial multicenter international experience with a novel portable cryoballoon ablation system (with video). Gastrointest Endosc 2018; 87: 574-581

[5] van Munster SN, Overwater A, Haidry R et al. Focal cryoballoon versus radiofrequency ablation of dysplastic Barrett's esophagus: impact on treatment response and postprocedural pain. Gastrointest Endosc 2018; 88: 795-803 e2

[6] Kunzli HT, Scholvinck DW, Meijer SL et al. Efficacy of the CryoBalloon Focal Ablation System for the eradication of dysplastic Barrett's esophagus islands. Endoscopy 2017; 49: 169-175

[7] Scholvinck DW, Kunzli HT, Kestens C et al. Treatment of Barrett's esophagus with a novel focal cryoablation device: a safety and feasibility study. Endoscopy 2015; 47: 1106-1112

[8] van Munster SN, Pouw RE, Bergman J. Randomized studies for Barrett's ablation: just because we can doesn't mean we should. Gastrointest Endosc 2019; 89: 690-692 